\title{
Spatial distribution of heat flux and fluctuations in turbulent Rayleigh-Bénard convection
}

\author{
Rajaram Lakkaraju, ${ }^{1}$ Richard J. A. M. Stevens, ${ }^{1,2}$ Roberto Verzicco ${ }^{1,3}$ Siegfried Grossmann, ${ }^{4}$ Andrea Prosperetti,${ }^{1,2}$ \\ Chao Sun, ${ }^{1}$ and Detlef Lohse ${ }^{1}$ \\ ${ }^{1}$ Faculty of Science and Technology, Mesa + Institute and J. M. Burgers Center for Fluid Dynamics, University of Twente, \\ 7500AE Enschede, The Netherlands \\ ${ }^{2}$ Department of Mechanical Engineering, Johns Hopkins University, Baltimore, Maryland 21218, USA \\ ${ }^{3}$ Department of Mechanical Engineering, University of Rome "Tor Vergata”, Via del Politecnico 1, 00133 Rome, Italy \\ ${ }^{4}$ Department of Physics, University of Marburg, Renthof 6, D-35032 Marburg, Germany \\ (Received 5 November 2011; revised manuscript received 20 August 2012; published 26 November 2012)
}

\begin{abstract}
We numerically investigate the radial dependence of the velocity and temperature fluctuations and of the time-averaged heat flux $\bar{j}(r)$ in a cylindrical Rayleigh-Bénard cell with aspect ratio $\Gamma=1$ for Rayleigh numbers Ra between $2 \times 10^{6}$ and $2 \times 10^{9}$ at a fixed Prandtl number $\operatorname{Pr}=5.2$. The numerical results reveal that the heat flux close to the sidewall is larger than in the center and that, just as the global heat transport, it has an effective power law dependence on the Rayleigh number, $\bar{j}(r) \propto \mathrm{Ra}^{\gamma_{j}(r)}$. The scaling exponent $\gamma_{j}(r)$ decreases monotonically from 0.43 near the axis $(r \approx 0)$ to 0.29 close to the sidewalls $(r \approx D / 2)$. The effective exponents near the axis and the sidewall agree well with the measurements of Shang et al. [Phys. Rev. Lett. 100, 244503 (2008)] and the predictions of Grossmann and Lohse [Phys. Fluids 16, 1070 (2004)]. Extrapolating our results to large Rayleigh number would imply a crossover at $\mathrm{Ra} \approx 10^{15}$, where the heat flux near the axis would begin to dominate. In addition, we find that the local heat flux is more than twice as high at the location where warm or cold plumes go up or down than in plume depleted regions.
\end{abstract}

DOI: 10.1103/PhysRevE.86.056315

PACS number(s): 47.55.pb, 44.25.+f

\section{INTRODUCTION}

In Rayleigh-Bénard (RB) convection a fluid is heated from below and cooled from above. This problem of thermal convection is of the utmost importance from an applied point of view. Examples are thermal convection in the atmosphere, in the oceans, and in process technology. For recent reviews of RB convection we refer to Refs. [1,2].

In a cylindrical container, the dynamics of a RB system depends on three control parameters, the Rayleigh number $\mathrm{Ra}=$ $g \beta \Delta L^{3} / \nu \kappa$, the Prandtl number $\operatorname{Pr}=\nu / \kappa$, and the aspect ratio $\Gamma=D / L$. Here $g$ is the gravitational acceleration, $\beta$ the isobaric thermal expansion coefficient, $\Delta$ the temperature difference between the top and bottom plates, $v$ the kinematic viscosity, $\kappa$ the thermal diffusivity, and $L$ and $D$ are the height and diameter of the cylinder. The response of the system is expressed by the Nusselt number $\mathrm{Nu}$, the dimensionless heat flux [1].

Previous studies mainly focused on determining the global heat flux as a function of $\mathrm{Ra}$ and $\operatorname{Pr}$. For water $(\mathrm{Pr}=2-7)$, in the experimentally available range of $\mathrm{Ra}=10^{8}-10^{11}$, one finds that the global heat transport effectively scales as $\mathrm{Nu} \sim$ $\mathrm{Ra}^{0.29-0.31}[1,3-8]$. The effective exponents for the global heat flux are well described by the unifying theory of Refs. [4,9-11]. That theory also made predictions for the scaling exponents of the local heat flux in the center of the cell and at the sidewall [11]. The reasoning is based on splitting the thermal energy dissipation field into its plume and background contributions; similarly the kinetic energy dissipation is decomposed into its boundary layer and bulk contributions. By doing this Grossmann and Lohse [11] accounted for the various scalings in the Ra-Pr parameter space. They found that the local heat flux has an effective power law dependence on the Ra number, $\bar{j}(r) \propto \mathrm{Ra}^{\gamma_{j}(r)}$, and obtained a prediction for the scaling exponent $\gamma_{j}=0.45$ in the bulk (center) and $\gamma_{j}=0.22$ at the plume (sidewall) regions.
In order to understand the heat flux one has to either rely on Eulerian [12] or Lagrangian [13,14] measurements where the complex interplay between velocity and temperature can be studied. Advancements in experimental techniques made it possible to measure the vertical local velocity $u_{z}(\mathbf{r}, t)$ and the local temperature $T(\mathbf{r}, t)$ at a given spatial location $\mathbf{r}$ as functions of time. This allowed Shang et al. $[12,15,16]$ to determine the local convective heat flux [17]

$$
j(\mathbf{r}, t)=\frac{u_{z}(\mathbf{r}, t)\left[T(\mathbf{r}, t)-T_{0}\right]}{\kappa \Delta / L},
$$

where $T_{0}$ is the mean bulk temperature. They determined the probability density functions (PDF) of the local heat flux in the axis and sidewall regions and showed that the vertical heat flux is highly non-Gaussian and intermittent due to thermal plumes. This work stimulated Ching et al. [18] to theoretically study the problem. They decomposed the velocity field into a part associated with strong temperature fluctuations plus a background and found that, with the definitions they used, the local heat transport associated with the former velocity component near the axis of the cell scaled as $\mathrm{Ra}^{1 / 7}$. Later experiments by Shang et al. [16] revealed that the effective scaling exponent for the local heat flux is about 0.49 near the cell axis and about 0.24 near the sidewall, which confirms the main results obtained by Grossmann and Lohse [11], but is in disagreement with the model of Ching et al. [18].

In experiments it is quite difficult to measure the heat flux at many points in the cell due to problems with measurement techniques and the presence of inherent noise levels. The measurements at just one or two points may not be enough to understand the complex dynamics involved in the convection process. The present paper offers numerical results which complement the work initiated by Shang et al. $[12,16]$. We provide information on the heat flux at one-quarter, one-half, 
and three-quarters of the cell height for several radial positions $r$, not only near the axis and the sidewall. This information allows us to understand the two limits in the unifying theory [11] on the effective scaling exponents, one valid in the bulk (the central region of the cell) and the other valid in the plume region. As a result we clearly see persistence of the inhomogeneous nature of the flow in the radial direction which leads to different scaling exponents.

In experiments the local velocity is measured by LDV/PIV techniques at a spatial position which slightly differs from the location of the local temperature measurement. This spatial misalignment may possibly affect the results. In numerical simulations, in contrast, one has all the information on the flow field and thus the local heat flux can be calculated from the velocity and temperature measurements at exactly the same position. Finally, we comment on the local heat flux distribution with respect to the large scale circulation and the ultimate regime mentioned in Refs. $[9,11,19]$ and provide data to illuminate the differences between the measurements of Shang et al. [16] and the earlier Ching et al. [18] claims.

In simulations the Ra number range and the duration available for time averaging are more limited than in experiments. In order to mitigate the latter shortcoming we have limited the $\mathrm{Ra}$ number of our simulations. In the next section we briefly describe the numerical procedure before discussing our results on the local heat flux and the velocity and temperature fluctuations.

\section{NUMERICAL METHOD}

We performed direct numerical simulations for a Boussinesq fluid in a unit aspect ratio $(\Gamma=1)$ cylinder with constant temperatures applied at the top and bottom plates and an adiabatic sidewall. The fluid simulated in our calculations is water at $32^{\circ} \mathrm{C}(\mathrm{Pr}=5.2)$ for $2 \times 10^{6} \leqslant \mathrm{Ra} \leqslant 2 \times 10^{9}$. The governing equations for momentum, energy, and mass conservation in dimensionless form are given by (see e.g. Ref. [20])

$$
\begin{aligned}
& \frac{D \mathbf{u}}{D t}=-\nabla p+\theta \hat{\mathbf{z}}+\left(\frac{\mathrm{Pr}}{\mathrm{Ra}}\right)^{1 / 2} \nabla^{2} \mathbf{u}, \\
& \frac{D \theta}{D t}=\frac{1}{(\mathrm{PrRa})^{1 / 2}} \nabla^{2} \theta, \quad \nabla \cdot \mathbf{u}=0 .
\end{aligned}
$$

Here the dimensionless variables are the velocity $\mathbf{u}$, scaled temperature $\theta$, and pressure $p$ (minus the hydrostatic contribution). The material derivative is denoted by $D / D t$. The unit vector $\hat{\mathbf{z}}$ is in the direction opposite to gravity. The physical variables length and velocity are made nondimensional by the cylinder height $(L)$, and the freefall velocity $U=\sqrt{g \beta \Delta L}$. Constant dimensionless temperatures of 1 and 0 are applied at the bottom and top plate, respectively.

The governing equations are solved on a staggered grid with second-order accuracy in space and time. For the time advancement a third-order Runge-Kutta scheme is used. This method is stable for a CFL number up to $\sqrt{3}$ [21] and here we used a CFL number of 1.2. In addition, the maximum time step of integration is restricted to 0.01 free-fall times. More details about the numerical method can be found in Refs. [20,22,23]. For the spatial resolution we followed the criteria set by Stevens et al. [24].

The code has been validated by effecting many comparisons of the calculated Nusselt number with experimental data showing agreement within $1 \%$ [24]. Recently, we have shown that statistical quantities (relative strength and temperature amplitudes of the large-scale circulation, and sidewall temperature gradient) measured in experiments and simulations agree up to the statistical accuracy that can be obtained in simulations [25].

A summary of the simulation parameters is shown in Table I. The first and second columns are Ra and the number of grid nodes. The volume- and time-averaged global heat transport, i.e., the Nusselt number $\mathrm{Nu}=1+\sqrt{\operatorname{Ra} \operatorname{Pr}}\left[\overline{\left\langle u_{z} \theta\right\rangle_{V}}\right]$, is shown in the third column; here the overline denotes time averages and the angular brackets $\langle\cdot\rangle_{V}$ volume averages. The number of grid points used to resolve the thermal boundary layers is shown in the fourth column. In a RB cell with no slip velocity condition at the walls the dimensional thermal energy dissipation rate, $\varepsilon_{T}=\kappa \Delta^{2} L^{-2} \overline{\left\langle|\nabla \theta|^{2}\right\rangle_{V}}$, and kinetic energy dissipation rate $\varepsilon_{K}=v^{3} L^{-4} \operatorname{Pr}^{-2} \operatorname{Ra} \overline{\left\langle|\nabla \mathbf{u}|^{2}\right\rangle_{V}}$ satisfy exact relationships with $\mathrm{Nu}$ (see Refs. [1,26]), namely $\varepsilon_{T}=\kappa \Delta^{2} L^{-2} \mathrm{Nu}$ and $\varepsilon_{K}=v^{3} L^{-4} \operatorname{Pr}^{-2} \mathrm{Ra}(\mathrm{Nu}-1)$. In order to validate our simulations the obtained energy dissipation rates are compared with $\mathrm{Nu}$ in Table I. These ratios are near

TABLE I. Summary of simulation parameters: the number of grid points used in angular $\left(N_{\phi}\right)$, radial $\left(N_{r}\right)$, and axial directions $\left(N_{z}\right)$, volume and time averaged Nusselt number $(\mathrm{Nu})$, the number of grid points in the thermal boundary layer $\left(n_{\mathrm{bl}}\right)$, convergence of exact relations for $\varepsilon_{K}$ and $\varepsilon_{T}$, comparing maximum of grid spacing in angular $\left(\delta \phi_{m}\right)$ and axial $\left(\delta z_{m}\right)$ directions with the Kolmogorov length scale based on global kinetic energy dissipation rate $(\eta)$, and the averaging time considered for the simulations are shown. The reported times are always measured in the units of free-fall time and the lengths in terms of cylinder height. For statistical averages we discarded the initial 140 free-fall times, to

\begin{tabular}{|c|c|c|c|c|c|c|c|}
\hline $\mathrm{Ra}$ & $N_{\phi} \times N_{r} \times N_{z}$ & $\mathrm{Nu}$ & $n_{\mathrm{bl}}$ & $\frac{\varepsilon_{K}}{\frac{v^{3}}{L^{4}} \frac{\mathrm{Ra}^{2}}{\mathrm{Pr}^{2}}(\mathrm{Nu}-1)}$ & $\frac{\varepsilon_{T}}{\kappa \frac{\Delta^{2}}{L^{2}} \mathrm{Nu}}$ & $\delta \phi_{m}, \delta z_{m}, \eta\left(\times 10^{2}\right)$ & time in $L / U$ \\
\hline $2 \times 10^{6}$ & $193 \times 49 \times 129$ & 10.93 & 19 & 1.008 & 0.968 & $2.26,1.45,3.41$ & 4200 \\
\hline $1 \times 10^{7}$ & $257 \times 65 \times 193$ & 16.58 & 18 & 1.010 & 0.971 & $2.44,0.73,2.04$ & 3700 \\
\hline $2 \times 10^{7}$ & $257 \times 65 \times 193$ & 20.71 & 18 & 1.007 & 0.843 & $2.44,0.73,1.62$ & 3700 \\
\hline $6 \times 10^{7}$ & $321 \times 97 \times 239$ & 28.48 & 14 & 0.989 & 0.923 & $1.96,0.97,1.13$ & 3000 \\
\hline $1 \times 10^{8}$ & $385 \times 129 \times 257$ & 33.25 & 15 & 0.997 & 0.955 & $1.62,0.73,0.95$ & 2800 \\
\hline $2 \times 10^{8}$ & $385 \times 129 \times 257$ & 40.87 & 13 & 1.002 & 0.937 & $1.62,0.73,0.76$ & 2700 \\
\hline $5 \times 10^{8}$ & $513 \times 161 \times 321$ & 52.80 & 13 & 1.005 & 0.947 & $1.22,0.51,0.56$ & 1900 \\
\hline $2 \times 10^{9}$ & $769 \times 193 \times 385$ & 80.34 & 11 & 0.992 & 0.959 & $0.82,0.34,0.26$ & 2040 \\
\hline
\end{tabular}
prevent transient effects from contaminating the results. 


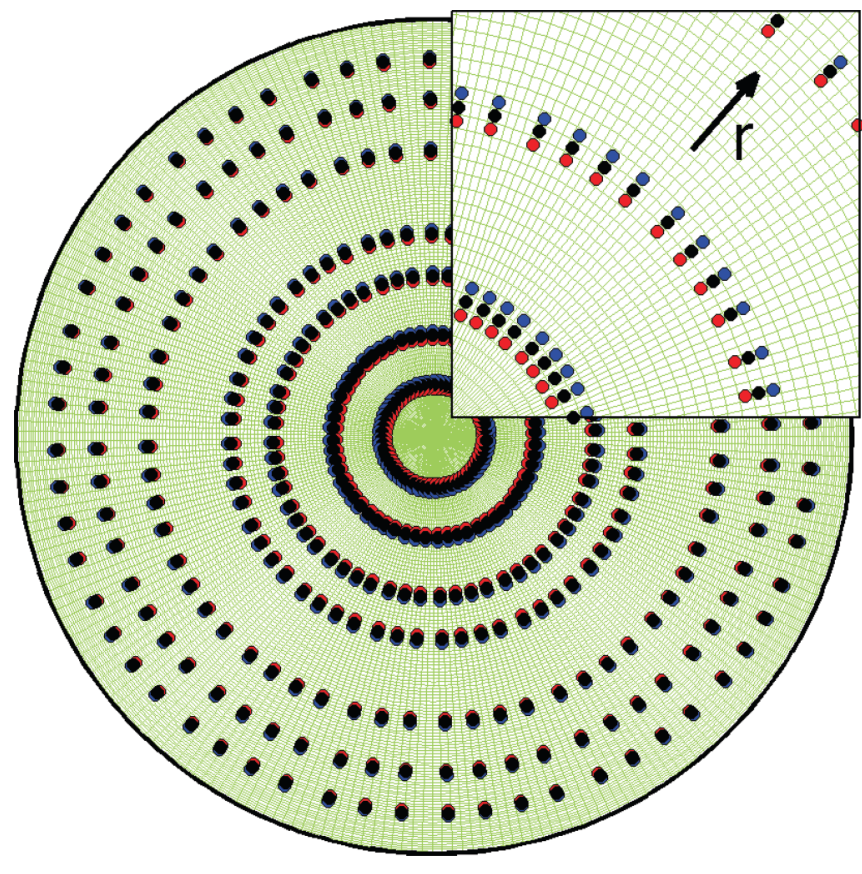

FIG. 1. (Color online) Computational grid used for $\mathrm{Ra}=2 \times$ $10^{7}$. We position numerical probes uniformly in the azimuthal direction in circles at seven radial locations $r / L=0.06,0.12,0.19$, $0.24,0.34,0.40,0.45$ (shown in black). For better statistics, on the mid-height plane, we use two more sets of probes [blue (outer), red (inner)] with an offset of one grid point. Inset shows a closeup view of the probe locations on the grid.

one, which proves the adequacy of the grid resolution. In the seventh column the largest grid spacings in the azimuthal and axial directions are compared with the Kolmogorov length. In the last column the total time used for the statistical averages is shown in free-fall times $(L / U)$. The total computational time was around $2.2 \times 10^{5}$ Power6 CPU hours. This large amount of time was needed to resolve the small scale motions and heat flux events.

We placed 1880 "numerical probes" at different radial locations on three different horizontal planes (at $z / L=0.25$, $z / L=0.50$, and $z / L=0.75)$, to obtain pointwise data on the temperature and vertical velocity in order to calculate the local heat flux according to Eq. (1). In each horizontal plane a number of azimuthally nearly equally spaced probes were placed on seven circles with radii $r / L=0.06,0.12$, $0.19,0.24,0.34,0.40$, and 0.45 ; see Fig. 1 . On each circle we distributed 60 probes at $z / L=0.50$ and 20 probes at $z / L=0.25,0.75$. In addition, on the mid-height plane, each circle was complemented by two other circles, one inside and one outside, spaced by one radial mesh length as shown in the upper right corner of Fig. 1. In total, we have information from 60 (azimuthal) $\times 7$ (radial) $\times 3($ sets $)=1260$ probes at the mid-height plane. For the planes at $z / L=0.25$ and at $z / L=0.75$ we have information from $20 \times 7=140$ probes.

\section{RESULTS}

\section{A. Local heat flux}

In Fig. 2 we show PDFs for the local instantaneous heat flux $j$ on the mid-height plane, at two different radial
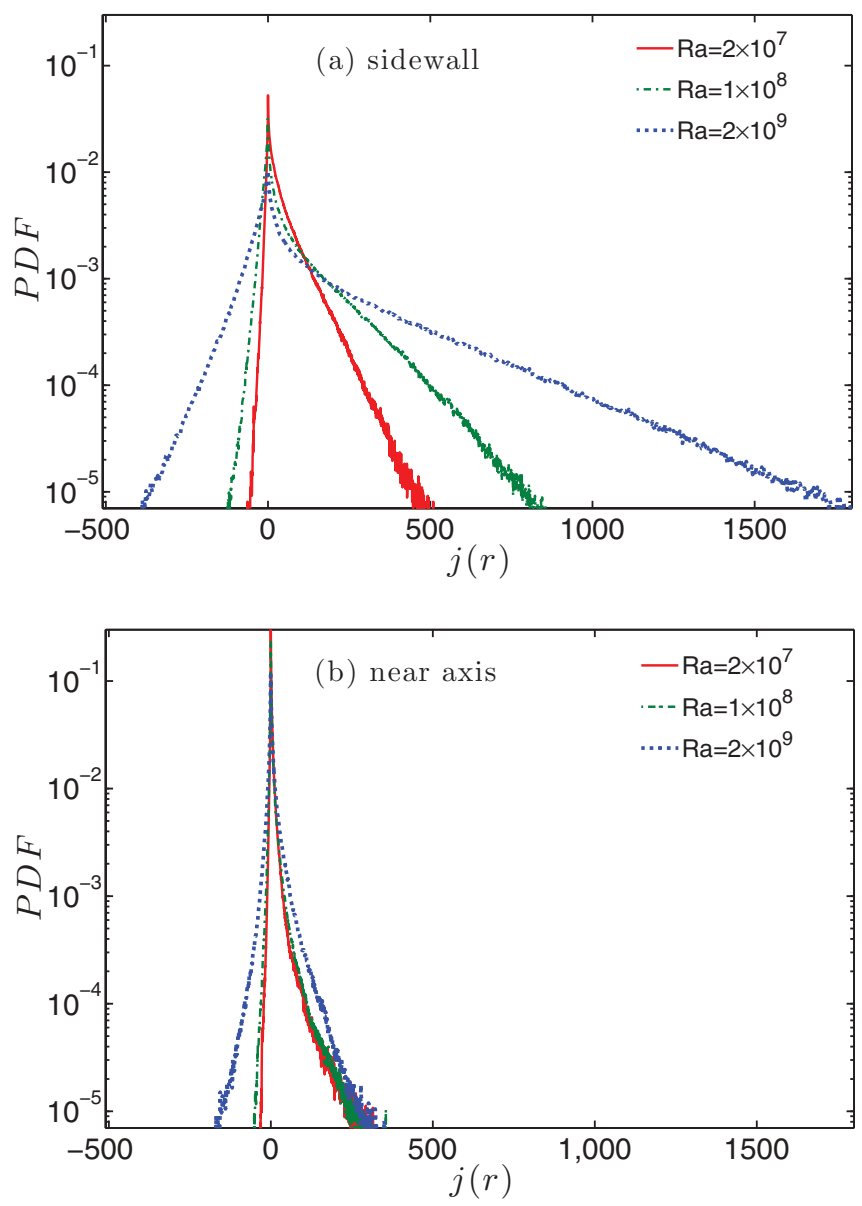

FIG. 2. (Color online) PDFs of the local instantaneous heat flux $j$ (a) near the sidewall at $r / L=0.45$ and (b) near the axis $r / L=0.06$ at midheight for $\mathrm{Ra}=2 \times 10^{7}$ (red solid), $1 \times 10^{8}$ (green dash-dot), and $2 \times 10^{9}$ (blue dash).

positions, one near the cell axis $(r / L=0.06)$ and one near the sidewall $(r / L=0.45)$. Note that the latter is outside the kinetic boundary layer (BL) which at this Prandtl number has a thickness of $\lambda_{u} / L \approx 3.6 \mathrm{Ra}^{-0.26 \pm 0.03}$ [28]. According to this scaling law we have $\lambda_{u} / L \approx 0.05$ for $\mathrm{Ra}=10^{7}$ and $\lambda_{u} / L \approx 0.016$ for $\mathrm{Ra}=10^{9}$. From our numerical calculations of the kinetic sidewall BL thickness, as identified by the location of the maximal velocity fluctuations, we get even slightly smaller values. Obviously, there is no thermal BL at the sidewalls due to the adiabatic boundary conditions.

The first striking observation from Fig. 2 is that the absolute value of the heat flux is much larger at the side walls (a) as compared to the center (b). This is consistent with the expectation that most of the heat is transported by the large-scale convection roll and of course it is well known [16]. Near the sidewall we observe some events with a heat flux as high as 15 times the average. In this region, the PDF has a marked positive skewness due to rising and falling of the warm and cold plumes. The PDF of the local heat flux in the cell center also has a positive skewness, which indicates that plumes can travel through this region as well. Figure 3 shows examples of the time evolution of $j$ as a function of the dimensionless time $t$ close to the cell center $(r / L=0.06$, 


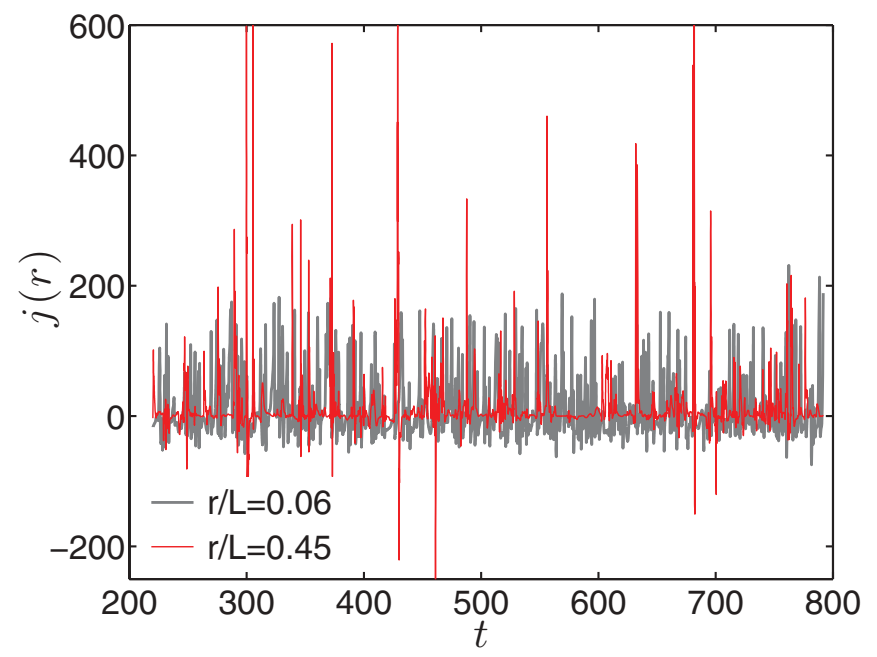

FIG. 3. (Color online) Time series for the heat flux near the sidewall (thin, red) and in the center (thick, gray) for $\mathrm{Ra}=1 \times 10^{8}$.

gray) and to the sidewall $(r / L=0.45$, red $)$ for $\mathrm{Ra}=10^{8}$ and again reflects the presence of much stronger heat transport events near the sidewall than near the axis.

One of the main features of turbulence is the small scale intermittency that is measured as departure from a Gaussian character of the PDF, mainly the tails and the peakedness. This can be quantified by calculating the flatness $F_{4}$ of the PDF. For strongly intermittent signals however the integral of $j^{4} \times \operatorname{PDF}(j)$ defining the flatness may not converge. To examine this issue we calculate the angular average of this quantity at $r / L=0.06$ and at $r / L=0.45$ on the mid-height plane. While at the sidewall this quantity decays for large $|j|$ sufficiently fast, see Fig. 4 (this feature is more evident when data is plotted on a linear rather than log scale), and thus permits the calculation of the flatness (showing strong intermittency, $F_{4} \approx 11$, inset of Fig. 4 ), in the center the inter-

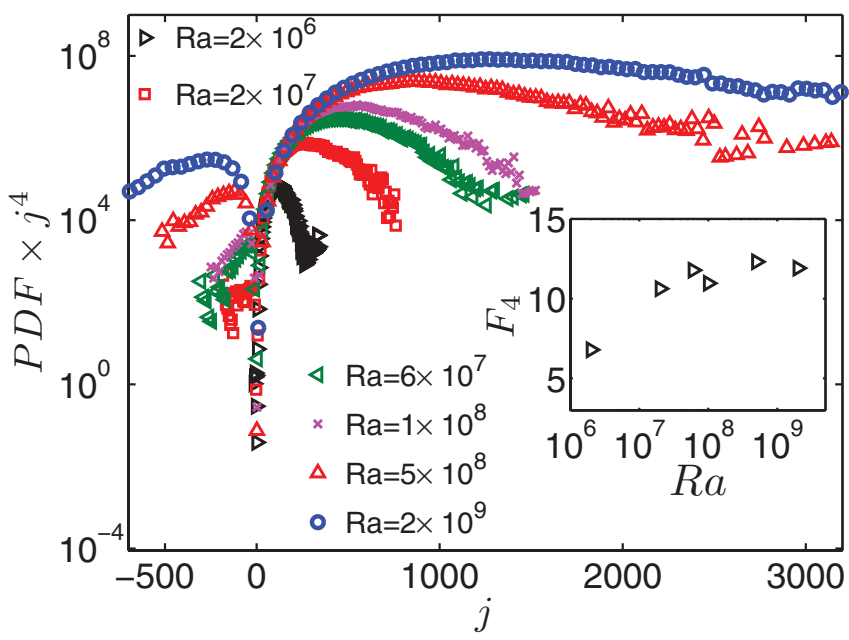

FIG. 4. (Color online) $j^{4} \times \operatorname{PDF}(j)$ vs $j$ is shown for different $\mathrm{Ra}$ near the sidewall region showing convergence of the flatness at that location. Note that the vertical axis is given in log scale, in contrast to the figures shown in the convergence test by Belin et al. [27], where $j^{4} \times \operatorname{PDF}(j)$ is given on a linear scale. The inset shows that the flatness $F_{4}$ increases as a function of Ra.
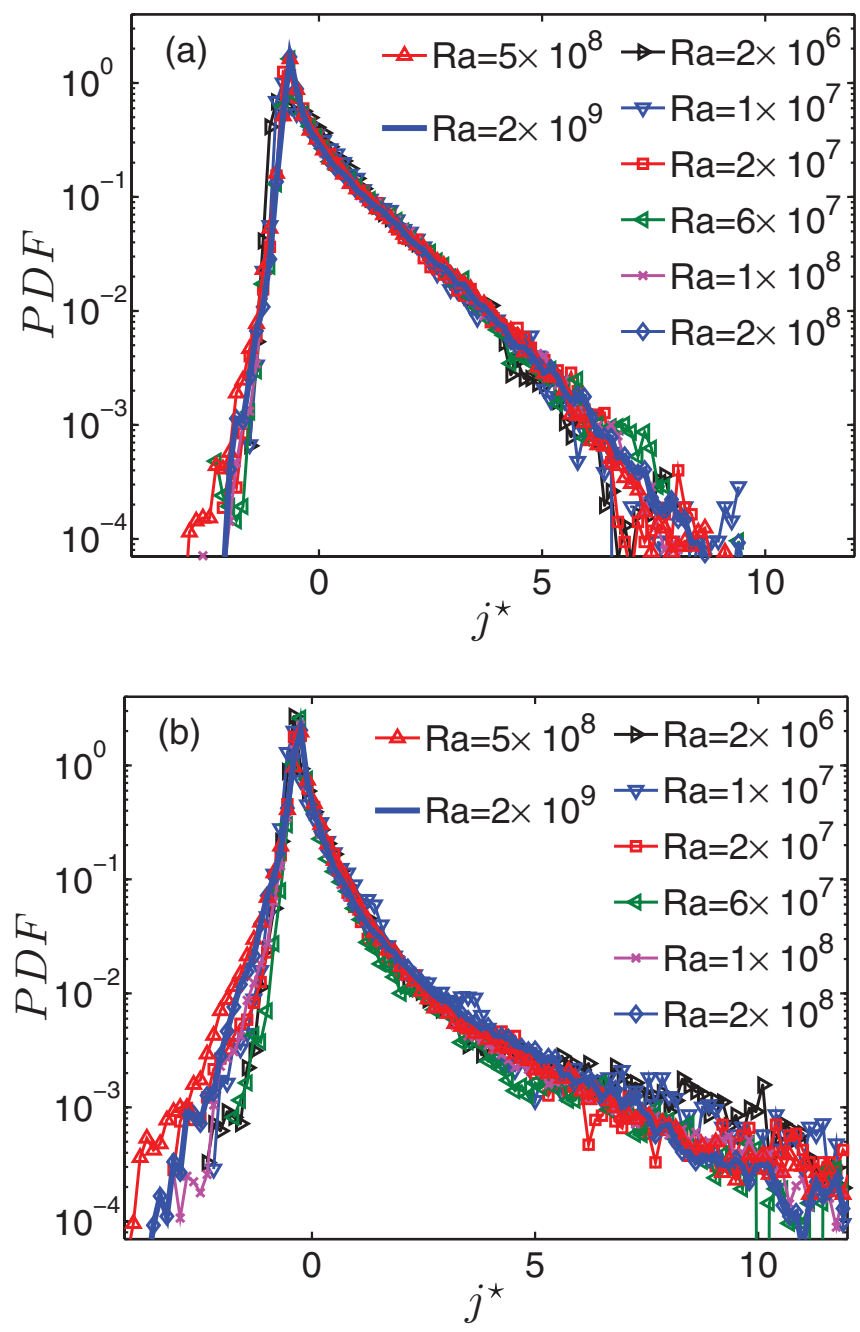

FIG. 5. (Color online) Centered PDFs of the normalized heat flux $j^{\star}(r)=[j(r)-\overline{j(r)}] / j_{\text {rms }}(r)$ on the mid-height plane (a) near the sidewall $r / L=0.45$, and (b) near the axis $r / L=0.06$.

mittency is so strong that no convergence for the flatness can be achieved.

By rescaling the heat flux $j$ with its standard deviation $j_{\text {rms }}$, the zero-mean PDF for the normalized heat flux $j^{\star} \equiv$ $(j-\bar{j}) / j_{\text {rms }}$ shows universality near the sidewall [see Fig. 5(a)]. The tails for the rescaled PDFs are shorter at the sidewalls compared to those at the center. This indicates relatively fewer plumes carrying a large heat flux at the sidewalls in contrast to relatively more plumes carrying a smaller heat flux at the center, and again underlines the extremely strong intermittency of the heat flux on the axis [see Fig. 5(b)].

Figure 6(a) shows the time- and angularly averaged heat flux as a function of $\mathrm{Ra}$ at different radial positions on the mid-height plane. The solid lines are the measurements of Shang et al. [16] taken right on the axis and very near to the wall. Figure 6(b) shows that the corresponding scaling exponent $\gamma_{j}$ as a function of the radial position decreases monotonically from 0.43 near the axis to 0.29 close to the sidewall. The measurements of Shang et al. [16] and the theoretical analysis of Grossmann and Lohse [11] only made statements on the values close to the sidewall and to the axis; these are well confirmed by the present results. In the Ra range 

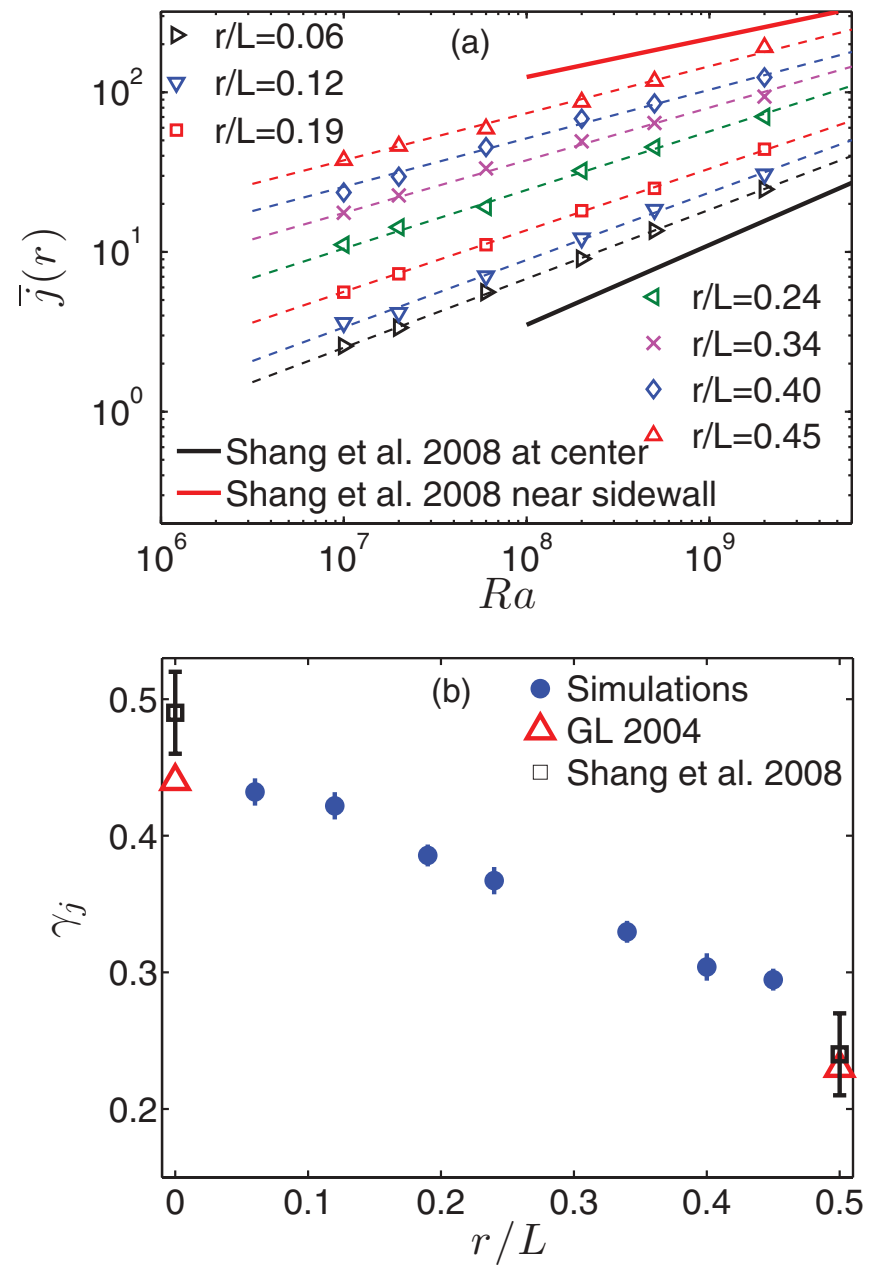

FIG. 6. (Color online) (a) Open symbols indicate the numerical results for the local heat flux averaged over time and angular position as function of Ra at different radial positions $r / L$. The solid lines show the experimental data of Shang et al. [16]. (b) The scaling exponent for the time- and angle-averaged heat flux as function of the radial position $r / L$ for the simulations, experiment [16], and theory [11].

considered here the heat transport near the sidewall is an order of magnitude larger than in the center. An extrapolation of the power law fits $\bar{j}=0.0025 \mathrm{Ra}^{0.43 \pm 0.01}$ obtained near the center and $\bar{j}=0.3236 \mathrm{Ra}^{0.29 \pm 0.01}$ valid near the wall shows that these become equal for $\mathrm{Ra} \approx 10^{15}$. This value is consistent with the prediction of the unifying theory [9]. Recent experiments [29] suggest the occurrence of this feature in the range $10^{13} \leqslant \mathrm{Ra} \leqslant 5 \times 10^{14}$, whereas Shang et al. [16] suggested that it happens at $\mathrm{Ra} \approx 10^{14}$, based on their extrapolation.

\section{B. Local fluctuations}

In this section, we determine the scaling with $\mathrm{Ra}$ of the velocity and temperature fluctuations with respect to their theoretical global mean at different radial locations.

Figure 7(a) shows $\operatorname{Re}_{\mathrm{rms}} \equiv \sqrt{(\mathrm{Ra} / \mathrm{Pr})} u_{\text {rms }}$ (equivalent to $U_{\mathrm{dim}} L / v$ with $U_{\mathrm{dim}}$ the dimensional rms velocity) as a function of Ra on the mid-height plane. For the normalized temperature fluctuations we take the root mean square of $\theta^{\prime}=\theta-1 / 2$.
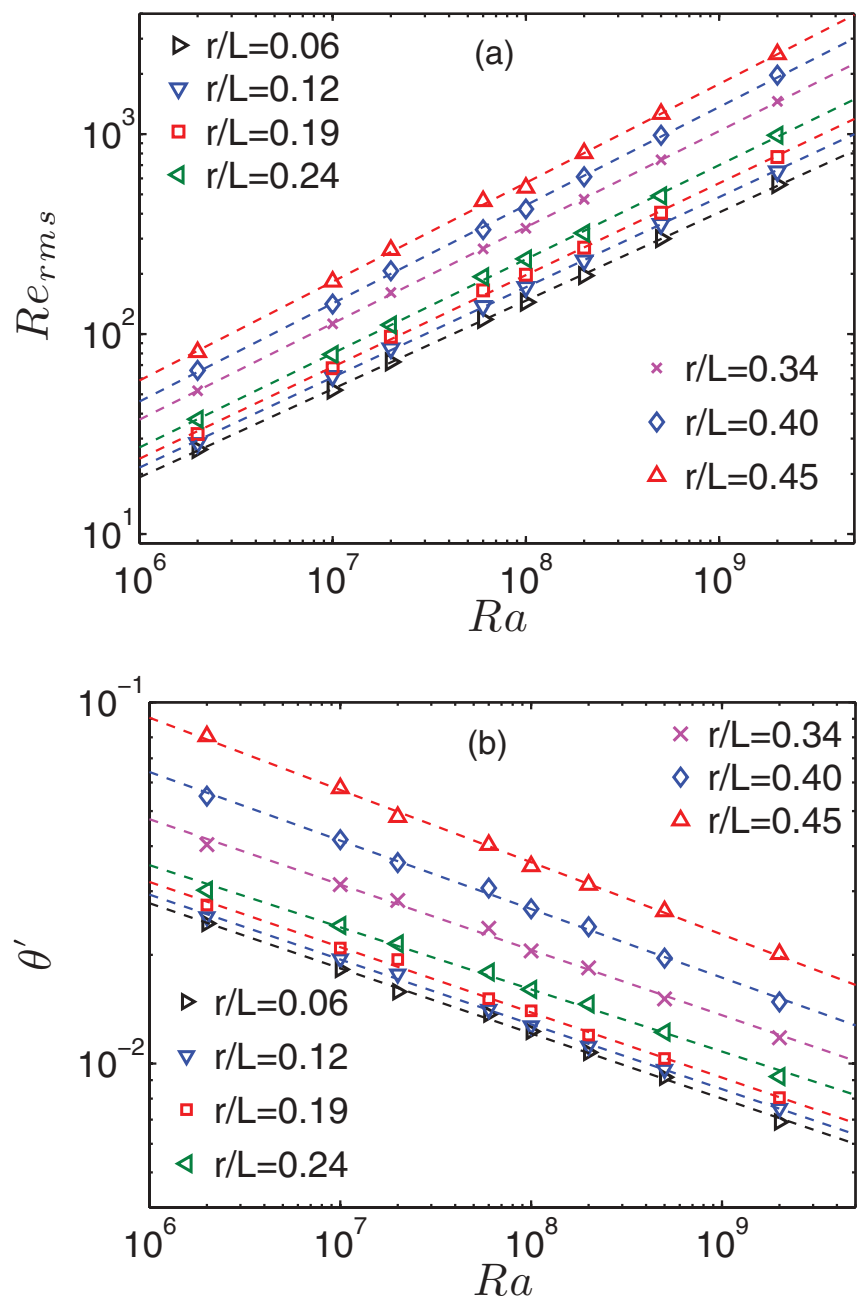

FIG. 7. (Color online) (a) Reynolds number based on the rms velocity $\mathrm{Re}_{\mathrm{rms}}$, and (b) the rms values of $\theta-1 / 2$, with $\theta$ the normalized temperature, as functions of Ra, both for different radial positions.

Figure 7(b) shows the results for this quantity versus Ra at different radial positions on the mid-height plane.

At all radial positions, fluctuations of both velocity and temperature exhibit a power law dependence on Ra proportional to $\mathrm{Ra}^{\gamma_{u}}$ and $\mathrm{Ra}^{\gamma_{\theta^{\prime}}}$, respectively. In this Ra number regime the thermal fluctuations close to the sidewall are an order of magnitude larger than at the cell center due to the plumes that travel along the wall.

Figure 8 shows that the corresponding velocity scaling exponents increase smoothly from $\gamma_{u}=0.44$ in the cell center to $\gamma_{u}=0.49$ near the sidewall. Figure 8 shows that the corresponding temperature scaling exponent decreases from $\gamma_{\theta^{\prime}}=-0.18$ in the cell center to $\gamma_{\theta^{\prime}}=-0.20$ near the sidewall.

Table II summarizes the data for the scaling exponents available in the literature and compares them with the present ones. There are some differences among the values reported. This is due in part to the spatial dependence of this quantity, shown in Fig. 8, but also to the use of different experimental techniques which measure somewhat different quantities. Overall, there is a general consistency among the data shown. 


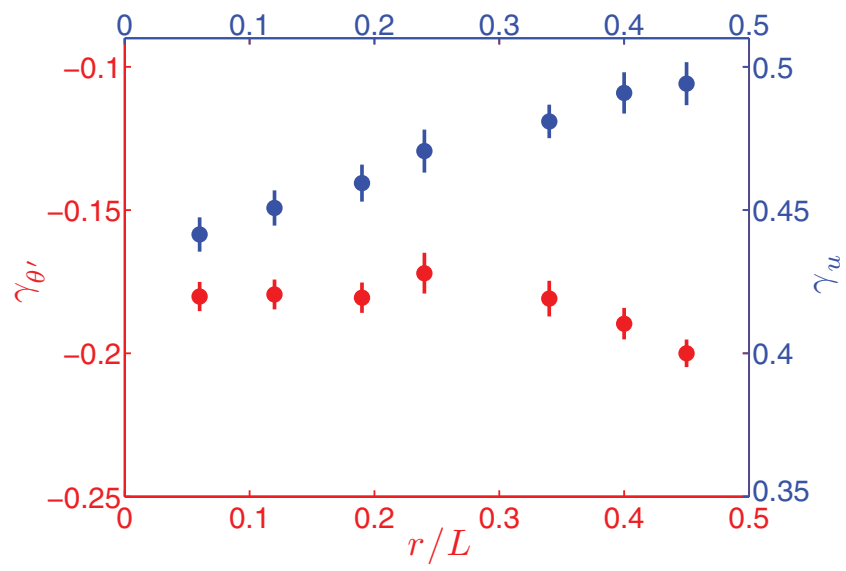

FIG. 8. (Color online) Variations in the velocity (blue, upper points and right axis) and temperature (red, lower point and left axis) scaling exponents with $\mathrm{Ra}$ are shown as functions of the radial position.

The origin of the residual differences cannot be ascertained on the basis of the presently available knowledge and must await further research.

The quantity $\operatorname{Re}_{\mathrm{rms}}$ shown in Fig. 7(a) would equal the Reynolds number based on the mean velocity fluctuation, $\operatorname{Re}^{\prime}=\sqrt{(\operatorname{Ra} / \operatorname{Pr})} u^{\prime}$, with $u^{\prime}=\sqrt{\overline{u_{z}^{2}}-\left(\overline{u_{z}}\right)^{2}}$ only for a perfectly converged simulation without any fixed large scale convection roll, for which $\overline{u_{z}}=0$. The simulation time necessary for this full convergence is completely outside the realm of practical computations as it would require averaging over a time sufficiently long with respect to slow processes such as the reorientation of the large scale circulation (see, e.g., Ref. [43] and Sec. IV A below). It is also interesting therefore to present in Fig. 9 results for $\mathrm{Re}^{\prime}$ similar to those of Fig. 7(a) for $\mathrm{Re}_{\mathrm{rms}}$. The subtraction of $\overline{u_{z}}$ removes the effect of the slowly varying large scale circulation which dominates near the sidewall but is fairly inconsequential near the axis. Thus the same scaling found near the axis in Fig. 7(a), $\mathrm{Re}_{\mathrm{rms}} \sim \mathrm{Ra}^{0.44}$, becomes applicable over the entire cell as suggested by the solid line.

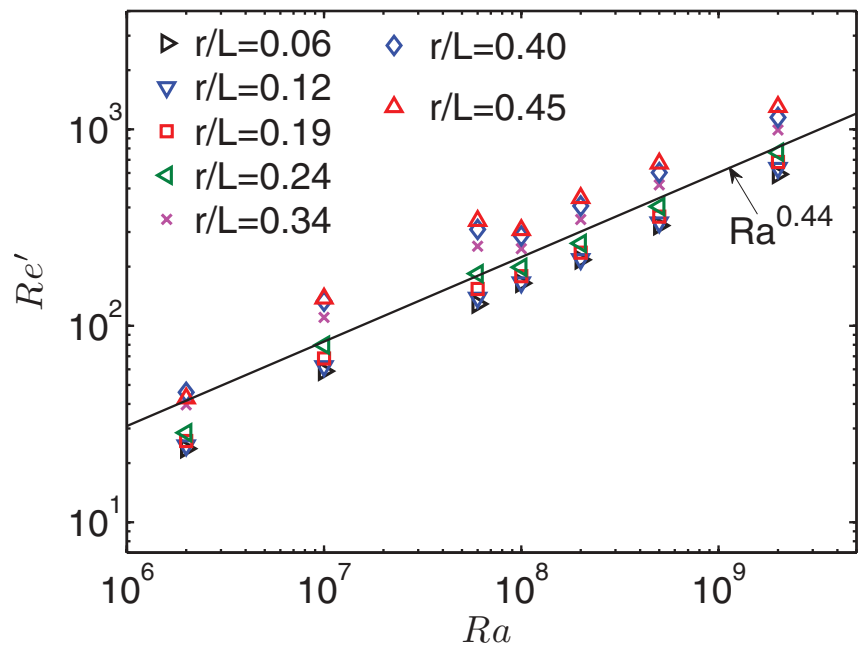

FIG. 9. (Color online) Reynolds number $\mathrm{Re}^{\prime}$ based on the rms velocity fluctuations vs $\mathrm{Ra}$ at different radial positions; the solid line has the slope 0.44 suggested by value of $\gamma_{u}$ at $r / L=0.06$ from Fig. 7(a).

\section{STATISTICS WITH RESPECT TO THE ORIENTATION OF THE LARGE SCALE CIRCULATION}

For $\Gamma=1$, the flow in the cell is characterized by a large scale circulation (LSC) [31,41-43] as sketched in Fig. 10. Most of the plumes travel in the LSC plane close to the sidewall. In experiments the LSC orientation can be detected with thermistors embedded in the sidewall [44] which measure relatively higher and lower temperatures in the regions of upflow and downflow [43]. Here we want to determine how the local heat flux depends on the location of measurement with respect to the LSC orientation plane.

\section{A. Determination of LSC orientation}

A well-tested method to determine the LSC orientation as a function of time is to fit a cosine to the azimuthal distribution of the vertical velocity or temperature profiles near the sidewalls [1,43-45]. For this purpose we use the

TABLE II. Summary of the velocity and temperature scaling exponents $\gamma_{u}$ and $\gamma_{\theta^{\prime}}$ reported in several experimental $(E)$ and theoretical $(T)$ studies. The experiments mentioned below have been carried out in cylindrical cells, unless stated otherwise.

\begin{tabular}{|c|c|c|c|c|c|c|c|}
\hline & $\mathrm{Ra}$ & $\operatorname{Pr}$ & $\Gamma$ & $\gamma_{u}$ (center) & $\gamma_{\theta^{\prime}}($ center $)$ & $\gamma_{u}($ sidewall $)$ & $\gamma_{\theta^{\prime}}($ sidewall $)$ \\
\hline Castaing et al. [30] (E) & $4 \times 10^{7}-6 \times 10^{12}$ & $0.65-1.5$ & 1 & $0.491 \pm 0.002$ & $-0.147 \pm 0.005$ & & \\
\hline Castaing et al. [30] (T) & $4 \times 10^{7}-6 \times 10^{12}$ & $0.65-1.5$ & 1 & $3 / 7$ & $-1 / 7$ & & \\
\hline Sano et al. [31] (E) & $10^{8}-10^{10}$ & $0.64-1.4$ & 1 & & & $0.485 \pm 0.005$ & \\
\hline Takeshita et al. [32] (E) & $10^{6}-10^{8}$ & 0.024 & 1 & & & $0.46 \pm 0.02$ & \\
\hline Ashkenazi et al. $[33,34](E)$ & $10^{11}-5 \times 10^{14}$ & $27-190$ & 1 (square) & & & $0.43 \pm 0.02$ & \\
\hline Chavanne et al. [35] (E) & $10^{7}-6 \times 10^{12}$ & $0.7-4$ & 0.5 & & & 0.49 & \\
\hline Daya and Ecke [36] $(E)$ & $2 \times 10^{8}-4 \times 10^{9}$ & 5.46 & 0.79 & $0.5 \pm 0.03$ & $-0.10 \pm 0.02$ & & \\
\hline Niemela et al. [37] (E) & $15 \times 10^{6}-10^{13}$ & 0.7 & 1 & & & 0.5 & \\
\hline Qiu et al. [38,39] (E) & $10^{8}-10^{10}$ & $5.4-5.5$ & 1 & 0.55 & & 0.46 & \\
\hline Lam et al. [40] (E) & $10^{6}-10^{11}$ & $6-1027$ & $0.5-4.4$ & & & 0.495 (bottom) & \\
\hline Grossman and Lohse [11] (T) & $10^{6}-10^{14}$ & $\sim 0.1-10$ & 1 & 0.39 & -0.11 to -0.16 & & -0.09 to -0.11 \\
\hline Shang et al. [16] (E) & $10^{8}-10^{10}$ & 4.4 & 1 & $0.49 \pm 0.03$ & $-0.14 \pm 0.03$ & $0.46 \pm 0.03$ & $-0.24 \pm 0.03$ \\
\hline Present work & $2 \times 10^{6}-2 \times 10^{9}$ & 5.2 & 1 & $0.44 \pm 0.01$ & $-0.18 \pm 0.01$ & $0.49 \pm 0.01$ & $-0.20 \pm 0.01$ \\
\hline
\end{tabular}




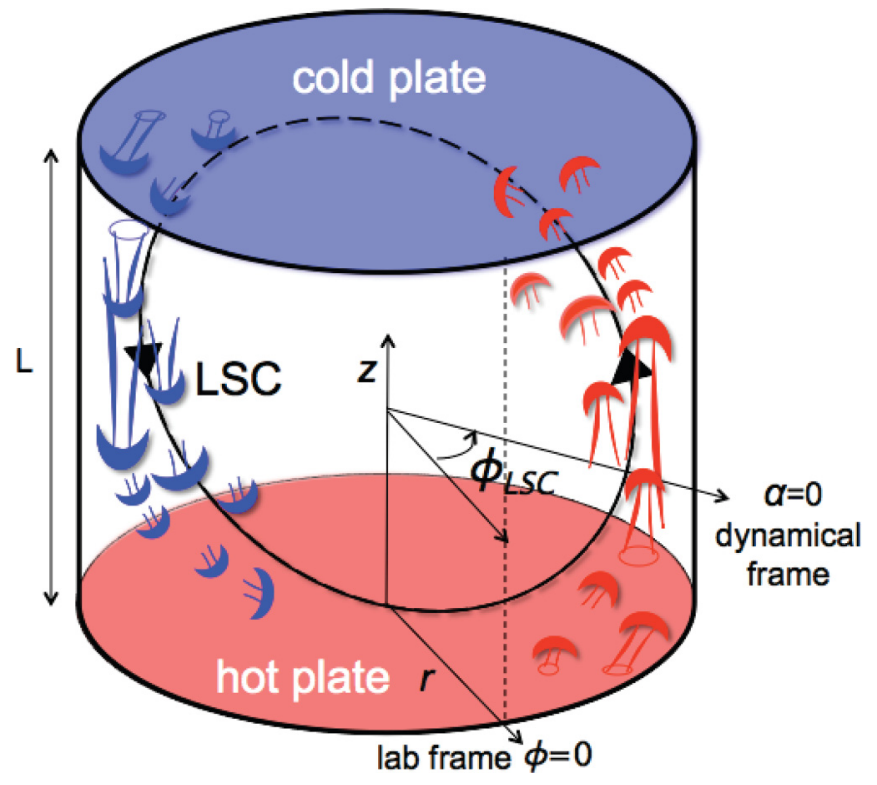

FIG. 10. (Color online) Sketch of the LSC in a cylindrical RB cell. The warm plumes go upwards on the right side, defined as $\alpha=0$, and go downwards on the left side of the cell, defined as $\alpha=\pi$. Due to the shape of the LSC the warm uprising fluid (cold down flowing fluid) is close to the sidewall at $z / L=0.25$ and $z / L=0.50(z / L=0.75$ and $z / L=0.50)$.

information from the numerical probes placed uniformly in the angular direction at $r / L=0.45$ (see Sec. II) [46]. To make sure that the LSC orientation is properly identified one has to avoid a determinant influence of individual plumes. This need requires that the instantaneous data be preprocessed by means of short-time moving averages. The choice of a proper averaging time depends on an order-of-magnitude estimation of the LSC circulation time. Such an estimate can be found by dividing the length of the longest path around the cell, $2 L+2 D=4 L$ for $\Gamma=1$, by an estimate of the fluid velocity. An upper limit is the free-fall velocity, with which we find $4 L / U=4$ free-fall times. A more realistic estimate can be found by using the computed velocity which, for $\mathrm{Ra}=10^{8}$, is about $0.15 \mathrm{U}$ [see Fig. 11(a)] and somewhat smaller for $\mathrm{Ra}=10^{9}$. With this estimate we find $\approx 27$ free-fall times. On this basis we decided to use four different short-time averaging durations, namely $4,10,20$, and 50 free-fall times. In this way we are more confident that the averaging time that we use covers the correct range.

We determine the LSC orientation by fitting the expression

$$
\left(u_{z}\right)_{i}=\left(u_{z}\right)_{m}+A_{u} \cos \left(\phi_{i}+\phi_{\mathrm{LSC}}\right)
$$

to the time-averaged vertical velocity data. Here $\left(u_{z}\right)_{i}$ is the short-time moving-averaged vertical velocity provided by the $i$-th probe at the angular position $\phi_{i},\left(u_{z}\right)_{m}$ is the mean value, $A_{u}$ is the amplitude, and $\phi_{\mathrm{LSC}}$ is the angular position of the LSC with respect to the reference frame of the computation.

Figure 11(a) shows an example of the azimuthal vertical velocity profile, and the corresponding cosine fit, using a shorttime averaging window of 20 free-fall times for $\mathrm{Ra}=2 \times 10^{8}$. The maximum of the curve is marked with a red cross and
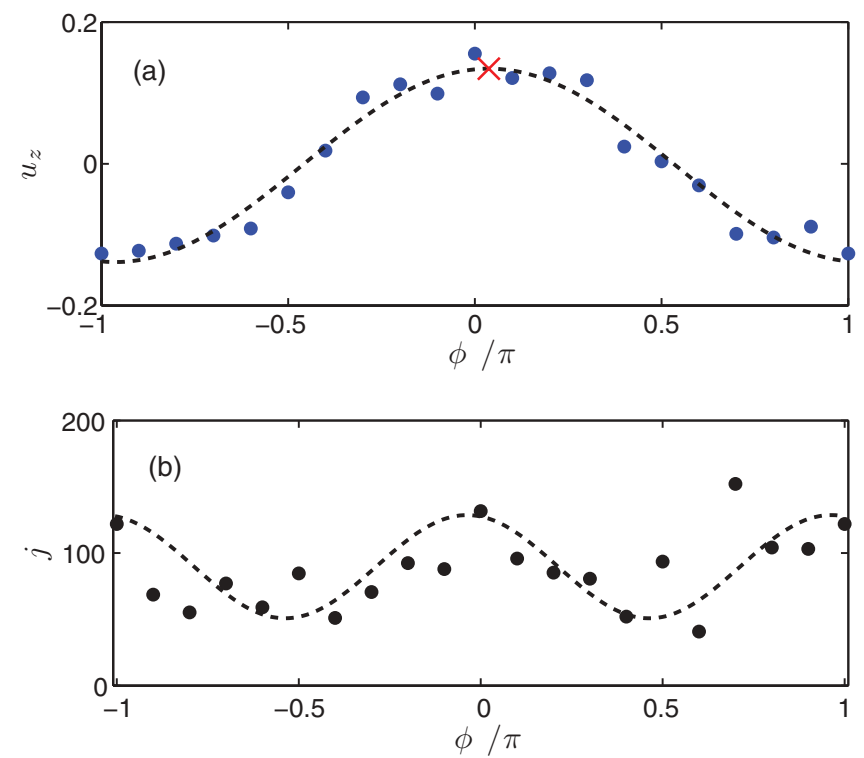

FIG. 11. (Color online) Angular dependencies of vertical velocity and heat flux on the mid-height plane at $r / L=0.45$ near the sidewall for $\mathrm{Ra}=2 \times 10^{8}$ : (a) vertical velocity (blue points) filtered by averaging of 20 free-fall times. The red cross indicates the maximum of the cosine fit, which we take as the LSC orientation. (b) Instantaneous local heat flux (black points). The black dash lines indicate the respective cosine fits to the data according to Eqs. (4) and (5).

identifies the position of the LSC plane. Figure 11(b) is an example of instantaneous heat flux data fitted as in Eq. (4), but with twice the frequency (see below),

$$
(j)_{i}=(j)_{m}+A_{j} \cos \left(2 \phi_{i}+\phi_{\mathrm{LSC}}\right) .
$$

Figure 12 shows $\phi_{\mathrm{LSC}}$ versus time as calculated using the four different averaging times and for four different Ra numbers. As expected, the fluctuations in the position of the LSC plane are somewhat greater when short averaging times are used. This figure clearly shows that the LSC orientation is different among simulations even though we used identical initial conditions for each simulation. We also note that the frequency of LSC reorientations observed in our simulations is roughly consistent with the experimental observations by Ref. [45].

Once the position of the LSC plane as a function of time has been determined, we can calculate the time-averaged vertical velocity $\bar{u}_{z}$ and heat flux $\bar{j}$ with respect to the LSC orientation. For this purpose we assign to the $u_{z}$ and $j$ instantaneously measured by the probe located at $\phi$ an angular position $\alpha=\phi-\phi_{\mathrm{LSC}}$ with respect to the LSC. We repeat this step for all the probes. In this way all the data of the numerical probes are converted from the computational to the LSC frame of reference.

Time averages in the LSC reference frame for the vertical velocity and for the heat flux at $r / L=0.45$ on the midheight plane are shown in Fig. 13. A comparison of the two panels in this figure shows that the heat flux has double the periodicity of the velocity, because the heat flux is enhanced in correspondence of both the upward and downward moving streams of the LSC. The local convective heat flux is lower 

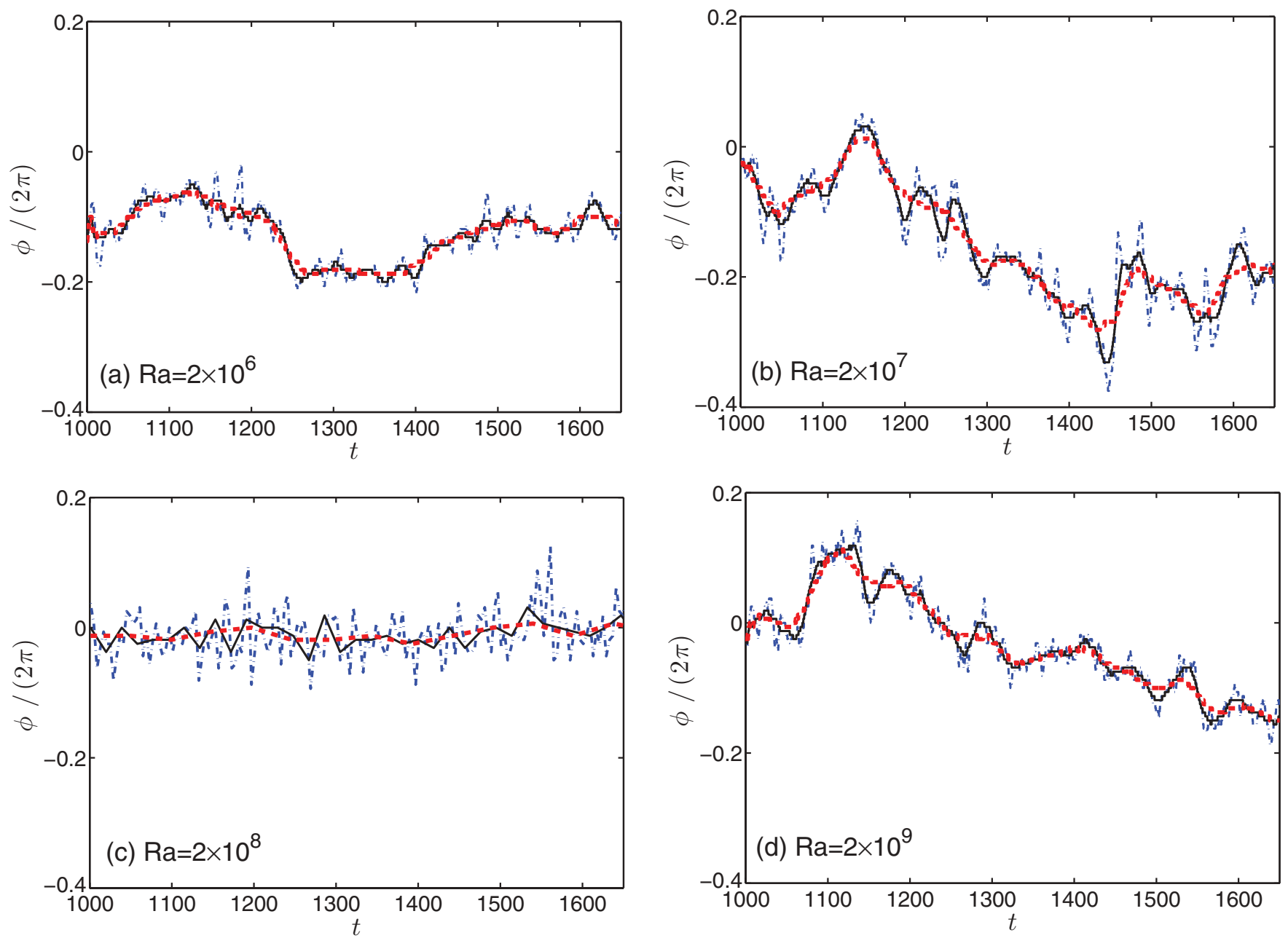

FIG. 12. (Color online) LSC orientation as function of time for different Ra. The time averaging used to determine the LSC orientation (see text) are 4, 20, and 50 free-fall times. The time averaging that is applied to the signal before the analysis is 4 (blue dash-dotted line), 20 (black solid), and 50 (red dash) free-fall times.

near $\alpha=\pi / 2$ and $3 \pi / 2$ where very few warm plumes ascend or cold plumes descend.

The four averaging times used to define $\phi_{\mathrm{LSC}}$ result, in principle, in four different values of $\alpha$ attributed to each probe reading. Therefore, for the $\alpha$ dependence of, for example, the average heat flux $\bar{j}(\alpha)$, one can draw four different curves. These four results are shown together by four sets of different symbols in the lower panel of Fig. 13 depicting the heat flux. Differences among these results are barely noticeable. This feature derives from the fact that each value of $j(\alpha)$ measured by each probe was assigned to one of 20 equal intervals in which the range of $\alpha$ was divided. Thus values falling within each $5 \%$ of the circle are attributed to the same value of $\alpha$. As can be seen in Fig. 12, most of the fluctuations of the LSC angular position fall within such a range irrespective of the averaging time. Figure 13 would not greatly change even if the angular intervals were, for example, halved. Now the values originally in one bin would be distributed between two adjacent smaller bins. However, by continuity, the resulting difference would not be large. This conclusion should be corrected if large fluctuations were frequent, which is by far not the case as Fig. 12 shows. Indeed, as seen in the figure, the short-time averages fluctuate very close to the long-time ones.

In Fig. 14 the amplitudes of the cosine fits for the axial velocity $A_{u}$, temperature $A_{\theta}$, and normalized heat flux $A_{j}$ [all defined by fits of Eqs. (4) and (5)], are shown as functions of $\mathrm{Ra}$ at midheight near the sidewall, $r / L=0.45$. Interestingly, these amplitudes have a power-law dependency on Ra, with scaling exponents $0.020 \pm 0.005,-0.250 \pm 0.010$, and $0.019 \pm 0.005$ for axial velocity, temperature, and local heat flux, respectively.

Figure 15(a) shows the time-averaged local heat flux as a function of $\mathrm{Ra}$ at different $\alpha$ at midheight near the sidewall. From the figure it is clear that this quantity has a power law dependence on $\mathrm{Ra}$. In agreement with the data in Fig. 14, we find that the local heat flux in the LSC plane increases faster than in the regions where few warm plumes rise and cold ones fall. This is also revealed when the local heat flux scaling exponent as function of $\alpha$, see Fig. 15(b), is considered.

Figure 16 shows the variation of the time-averaged local heat flux with $\alpha$ at three different heights. For $z / L=0.25$ larger values of $j$ occur near $\alpha=0$, and lower values near 

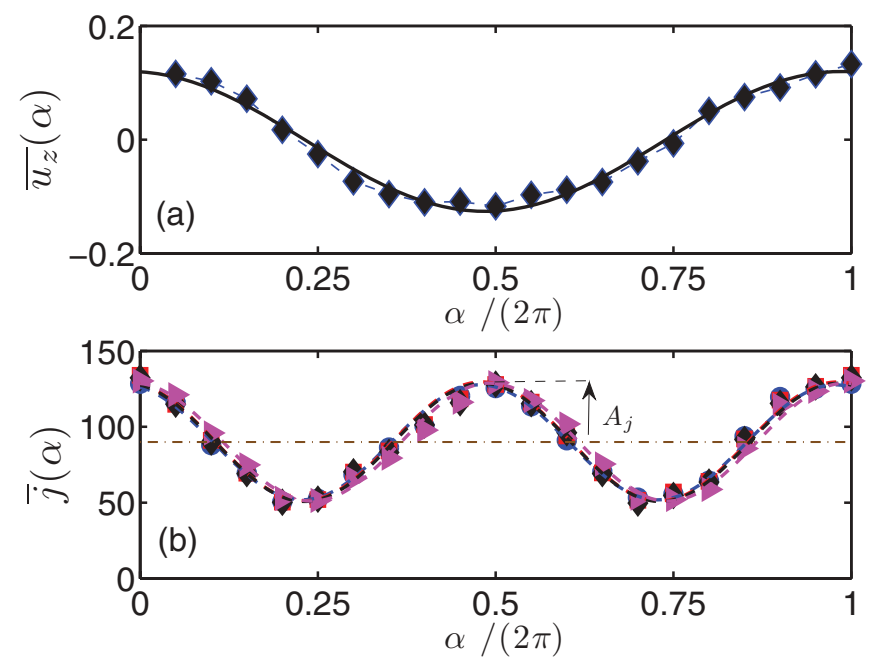

FIG. 13. (Color online) Time averaged quantities relative to the orientation of the LSC plane near the sidewalls $(r / L=0.45)$ at midheight: (a) vertical velocity $\overline{u_{z}}(\alpha)$; (b) local heat flux $\bar{j}(\alpha)$. Here the circles (blue), squares (red), diamonds (black), and triangles (magenta) correspond to time averages over 4, 10, 20, and 50 free-fall times, respectively. The black lines show the cosine fits according to Eqs. (4) and (5). The mean heat flux (thin-dash dot) and the amplitude of variation $\left(A_{j}\right)$ are also shown.

$\alpha=\pi$. A similar picture shifted by $\pi$ is found for $z / L=$ 0.75 , with higher values near $\alpha=\pi$ and lower values near $\alpha=0$. At $z / L=0.50$, on the other hand, the levels at $\alpha=0$ and $\alpha=\pi$ are comparable. These results suggest that the plane of the LSC is somewhat tilted with respect to the vertical.

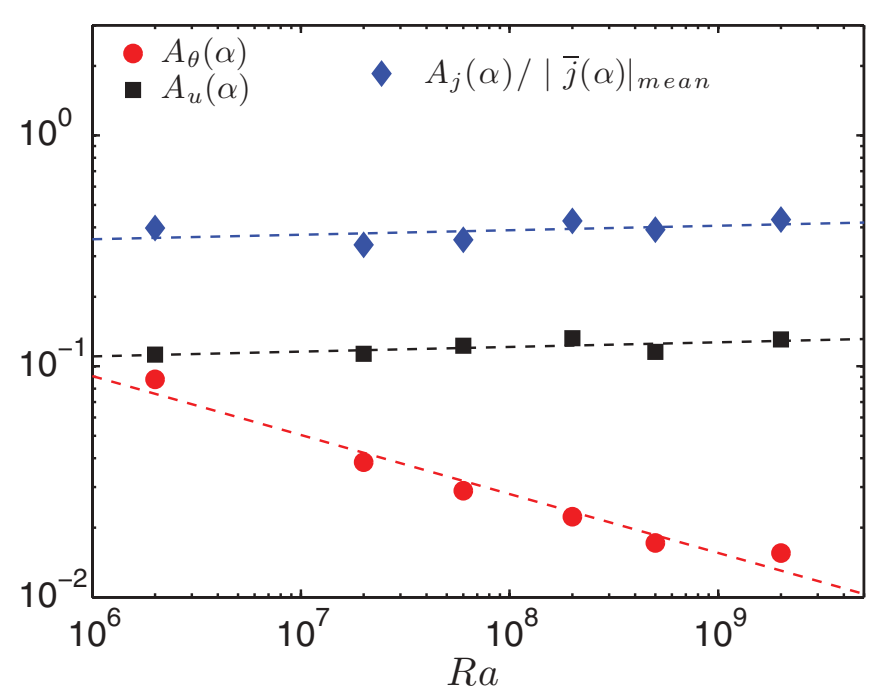

FIG. 14. (Color online) Amplitude variation as function of Ra of cosine fits near the sidewalls, $r / L=0.45$, at the midheight as function of Ra. The data are shown for temperature (circle, red), axial velocity (square, black), and normalized heat flux (diamond, blue). Here $|\bar{j}(\alpha)|_{\text {mean }}$ is the arithmetic mean heat flux in the azimuthal direction for a given $\mathrm{Ra}$. The dashed lines indicate the power law fits for the data.
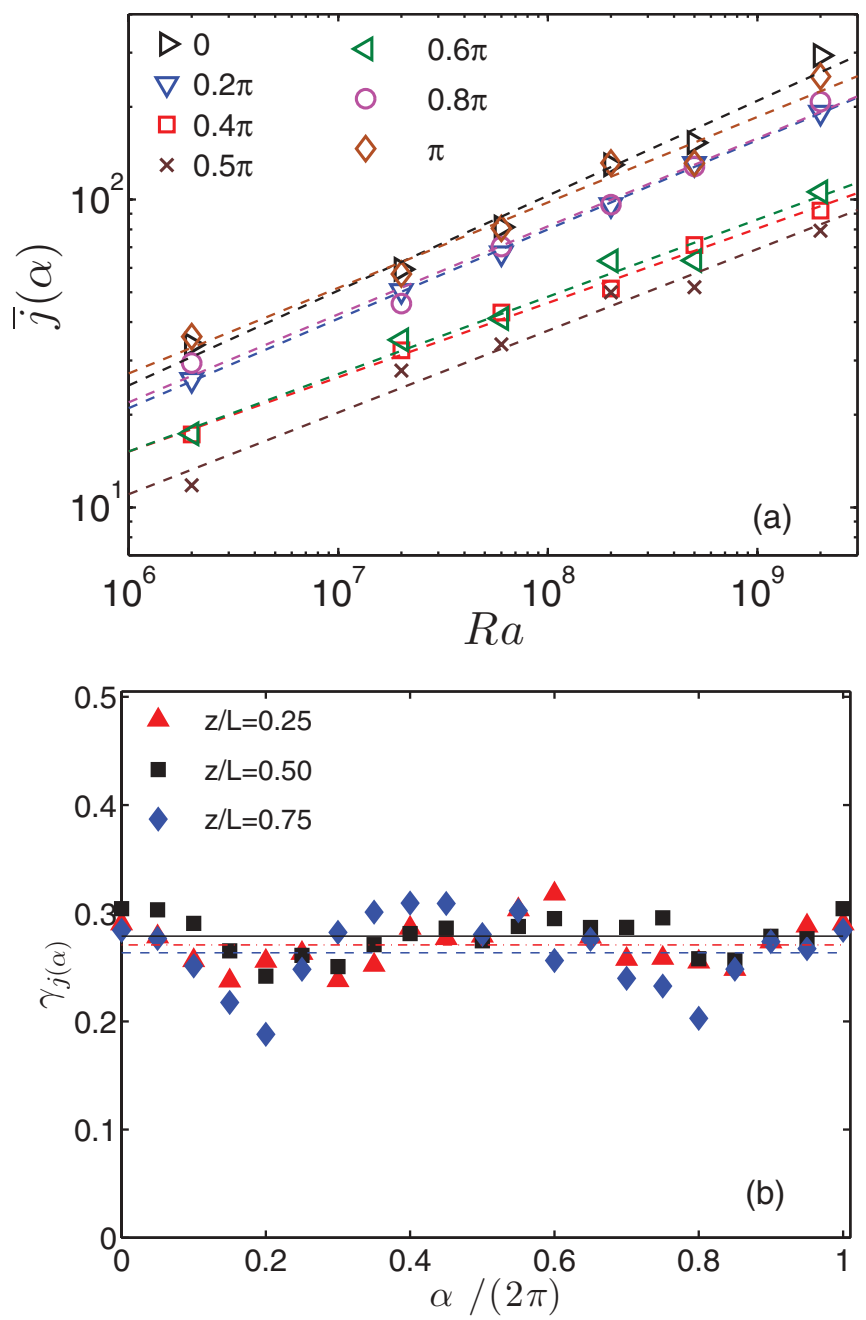

FIG. 15. (Color online) (a) Scaling of the time averaged local heat flux with Ra near the sidewalls, $r / L=0.45$, at midheight. The symbols indicate measurements taken at different $\alpha$. The dashed lines are the power law fits to the data. (b) Scaling exponent for the heat flux $\gamma_{j}(\alpha)$ relative to the LSC plane near the sidewall at $z / L=0.25$ (triangles, red), $z / L=0.50$ (square, black), and $z / L=0.75$ (diamond, blue). The straight lines indicate the arithmetic mean values of the scaling exponents.

\section{SUMMARY AND CONCLUSIONS}

To summarize, we investigated numerically the scaling of the local heat flux in Rayleigh-Bénard convection of a fluid with $\operatorname{Pr}=5.2$ (appropriate for water at $32{ }^{\circ} \mathrm{C}$ ) for $2 \times 10^{6} \leqslant$ $\mathrm{Ra} \leqslant 2 \times 10^{9}$ in a unit aspect ratio cylinder. In this Ra number regime the local heat flux is larger close to the sidewall than on the axis. The local heat flux $u_{z}\left(T-T_{0}\right)$ is a positive quantity both when fluid warmer than the average temperature $T_{0}$ rises and fluid colder than $T_{0}$ sinks. The PDFs of the local heat flux have a positive skewness due to the dominance of plume transport in this Ra range. On the mid-height plane, the scaling exponents of the local heat flux with Ra near the axis and close to the sidewall agree well with the measurements of Shang et al. [16] and the predictions of Grossmann and Lohse [11]. Here we have shown that these scaling exponents decrease monotonically with position $r$ from 0.43 near the 


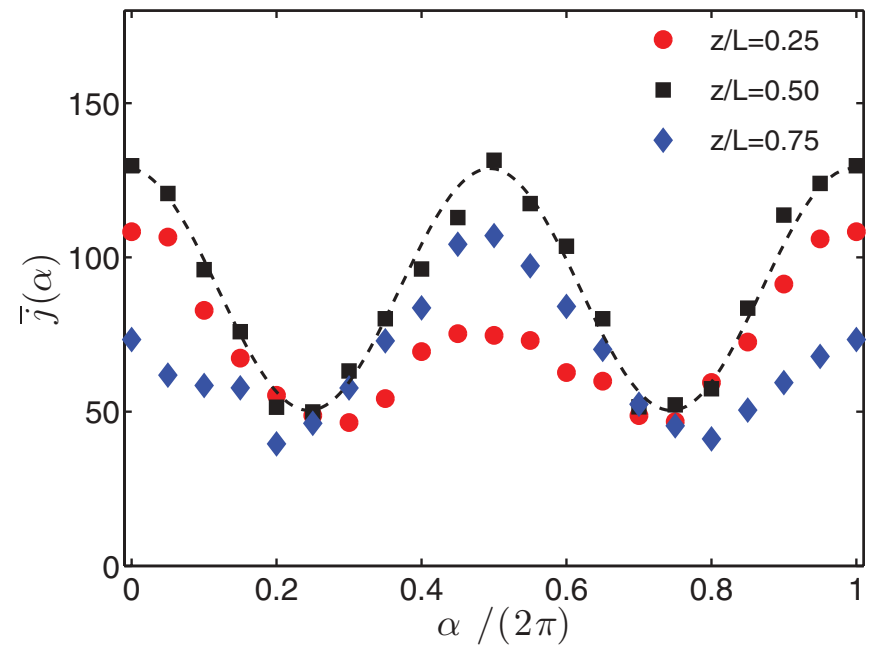

FIG. 16. (Color online) Time averaged local heat flux with respect to the LSC orientation for $\mathrm{Ra}=2 \times 10^{8}$ at different axial positions for $r / L=0.45$. The data are at the quarter height plane $z / L=0.25$ (circle, red), at midheight $z / L=0.50$ (square, black), and at the three-quarters height $z / L=0.75$ (diamond, blue). Moving averages are taken over 20 free-fall times. axis to 0.29 close to the sidewall. The scaling exponent for the Reynolds number based on the rms velocity depends on the radial position as well, with a value of 0.44 near the axis and 0.49 close to the sidewall. For the scaling exponents of the temperature fluctuations we find -0.18 and -0.20 , respectively. We showed the marked effect of the LSC which causes local heat fluxes more than twice as large in its plane than at $90^{\circ}$ from it. This effect becomes stronger for high Ra.

\section{ACKNOWLEDGMENTS}

We acknowledge the financial support by the Foundation for Fundamental Research on Matter (FOM) and the National Computing Facilities (NCF), both sponsored by NWO. This research is a part of industrial partnership program on Fundamentals of Heterogeneous Bubbly Flows (FHBF). The computations in this project have been performed on the Huygens cluster of SARA in Amsterdam.
[1] G. Ahlers, S. Grossmann, and D. Lohse, Rev. Mod. Phys. 81, 503 (2009).

[2] D. Lohse and K. Q. Xia, Annu. Rev. Fluid Mech. 42, 335 (2010).

[3] E. D. Siggia, Annu. Rev. Fluid Mech. 26, 137 (1994).

[4] S. Grossmann and D. Lohse, J. Fluid Mech. 407, 27 (2000).

[5] K.-Q. Xia, S. Lam, and S.-Q. Zhou, Phys. Rev. Lett. 88, 064501 (2002).

[6] A. Nikolaenko, E. Brown, D. Funfschilling, and G. Ahlers, J. Fluid Mech. 523, 251 (2005).

[7] D. Funfschilling, E. Brown, A. Nikolaenko, and G. Ahlers, J. Fluid Mech. 536, 145 (2005).

[8] C. Sun, L. Y. Ren, H. Song, and K. Q. Xia, J. Fluid Mech. 542, 165 (2005).

[9] S. Grossmann and D. Lohse, Phys. Rev. Lett. 86, 3316 (2001).

[10] S. Grossmann and D. Lohse, Phys. Rev. E 66, 016305 (2002).

[11] S. Grossmann and D. Lohse, Phys. Fluids 16, 4462 (2004).

[12] X.-D. Shang, X.-L. Qiu, P. Tong, and K.-Q. Xia, Phys. Rev. Lett. 90, 074501 (2003).

[13] Y. Gasteuil, W. L. Shew, M. Gibert, F. Chillá, B. Castaing, and J.-F. Pinton, Phys. Rev. Lett. 99, 234302 (2007).

[14] J. Schumacher, Phys. Rev. Lett. 100, 134502 (2008).

[15] X.-D. Shang, X.-L. Qiu, P. Tong, and K.-Q. Xia, Phys. Rev. E 70, 026308 (2004).

[16] X.-D. Shang, P. Tong, and K.-Q. Xia, Phys. Rev. Lett. 100, 244503 (2008).

[17] The diffusive heat flux $-\kappa \overline{\partial_{z} T(r, t)} /(\kappa \Delta / L)$ is less than $1 \%$ of the convective one, for all $r$ and Ra, due to the tiny temperature gradient at midheight.

[18] E. S. C. Ching, H. Guo, X. D. Shang, P. Tong, and K. Q. Xia, Phys. Rev. Lett. 93, 124501 (2004).

[19] D. Lohse and F. Toschi, Phys. Rev. Lett. 90, 034502 (2003).

[20] R. Verzicco and R. Camussi, J. Fluid Mech. 477, 19 (2003).
[21] P. Orlandi, Fluid Flow Phenomena: A Numerical Tool Kit (Kluwer Academic, Dordrecht, The Netherlands, 2001).

[22] R. Verzicco and P. Orlandi, J. Comput. Phys. 123, 402 (1996).

[23] R. Verzicco and R. Camussi, Phys. Fluids 9(5), 1287 (1997).

[24] R. J. A. M. Stevens, R. Verzicco, and D. Lohse, J. Fluid Mech. 643, 495 (2010).

[25] R. J. A. M. Stevens, H. J. H. Clercx, and D. Lohse, arXiv:1112.0411.

[26] B. I. Shraiman and E. D. Siggia, Phys. Rev. A 42, 3650 (1990).

[27] F. Belin, P. Tabeling, and H. Willaime, Physica D: Nonlin. Phenom. 93, 52 (1996).

[28] X. L. Qiu and K. Q. Xia, Phys. Rev. E 58, 486 (1998).

[29] X. He, D. Funfschilling, H. Nobach, E. Bodenschatz, and G. Ahlers, Phys. Rev. Lett. 108, 024502 (2012).

[30] B. Castaing, Gunaratne, G. Heslot, L. F. Kadanoff, A. Libchaber, A. S. Thomae, X.-Z. Wu, S. Zaleski, and G. Zanetti, J. Fluid Mech. 204, 1 (1989).

[31] M. Sano, X.-Z. Wu, and A. Libchaber, Phys. Rev. A 40, 6421 (1989).

[32] T. Takeshita, T. Segawa, J. A. Glazier, and M. Sano, Phys. Rev. Lett. 76, 1465 (1996).

[33] S. Ashkenazi and V. Steinberg, Phys. Rev. Lett. 83, 4760 (1999).

[34] S. Ashkenazi and V. Steinberg, Phys. Rev. Lett. 83, 3641 (1999).

[35] X. Chavanne, F. Chilla, B. Chabaud, B. Castaing, and B. Hebral, Phys. Fluids 13, 1300 (2001).

[36] Z. A. Daya and R. E. Ecke, Phys. Rev. Lett. 87, 184501 (2001).

[37] J. Niemela, L. Skrbek, K. R. Sreenivasan, and R. J. Donnelly, J. Fluid Mech. 449, 169 (2001).

[38] X.-L. Qiu and P. Tong, Phys. Rev. E 66, 026308 (2002).

[39] X.-L. Qiu, X.-L. Shang, P. Tong, and K. Q. Xia, Phys. Fluids 16, 412 (2004). 
[40] S. Lam, X.-D. Shang, S.-Q. Zhou, and K.-Q Xia, Phys. Rev. E 65, 066306 (2002).

[41] R. Krishnamurti and L. Howard, Proc. Natl. Acad. Sci. USA 78, 1981 (1981).

[42] K.-Q. Xia, C. Sun, and S.-Q. Zhou, Phys. Rev. E 68, 066303 (2003).
[43] E. Brown and G. Ahlers, J. Fluid Mech. 638, 383 (2009).

[44] E. Brown, A. Nikolaenko, and G. Ahlers, Phys. Rev. Lett. 95, 084503 (2005).

[45] E. Brown, and G. Ahlers, J. Fluid Mech. 568, 351 (2006).

[46] R. J. A. M. Stevens, H. J. H. Clercx, and D. Lohse, Phys. Fluids 23, 095110 (2011). 